Ann. Biol. anim. Bioch. Biophys., I970, 10 (4), 697-702.

\title{
INFLUENCE DE LA NATURE ET DU TAUX DES PROTÉINES ALIMENTAIRES SUR LA COMPOSITION EN ACIDES AMINÉS DU CONTENU DU CECUM DU RAT AXÉNIQUE
}

\author{
Étiennette COMBE, R. PION et E. SACQUET*
}

avec la collaboration technique d'Élisabeth Debras, Françoise Giovanni, G. Bayle, Catherine Mathis * et M. Lachkar *

Station d'Étude des Métabolismes,

Centre de Recherches de Clermont-Ferrand, 63-Saint-Genès-Champanelle Institut national de la Recherche agronomique

* Service des Animaux sans Germes,

Centre de Selection dos Animaux do Laboratoire., 78 - Gif-sur-Yvette

Centre national de la Recherche scientifique

La richesse en azote du régime mođifie considérablement les sécrétions digestives (TwombLY et MEYER, 1961) du rat de laboratoire élevé dans des conditions traditionnelles, en agissant tant sur la synthèse des enzymes que sur leur sécrétion ou leur vitesse d'inactivation (TwomвlySNOoK, I965). De plus, d'après CREAMER (I967), la vitesse de renouvellement des cellules épithéliales intestinales est diminuée en cas de diète totale (Stevens-Hooper, BlaIr, I958) ou de malnutrition protéique (DFO et RAMALingaswami, 1965) et les quantités d'azote endogène sécrétées dans le tube digestif s'en trouvent modifiées.

Ces phénomènes doivent se traduire chez le rat axénique dont le contenu digestif est à l'abri des attaques bactériennes par une modification qualitative et quantitative des matières azotées du contenu digestif ; c'est ce que nous avons voulu vérifier en analysant le contenu des cæcums de rats axéniques ayant reçu des aliments différents par leur teneur en matière azotée (tabl. I) et la nature de leurs protéines (tabl. 2), et en comparant certaines de leurs caractéristiques à celles de rats témoins " holoxéniques " (RAIBAUD $e t$ al., I966) ayant reçu les mêmes régimes (tabl. I).

Tous les régimes ont été stérilisés à l'autoclave à $\mathrm{I} 20^{\circ} \mathrm{C}$ pendant 20 minutes et distribués aux animaux pendant au moins 8 jours. La quantité moyenne de matière sèche ingérée par animal pendant les 5 jours précédant les prélèvements atteint environ $20 \mathrm{~g} / \mathrm{j}$ pour chaque lot d'animaux. Le poids moyen des rats (de race Wistar $\mathrm{CF}$ de Gif) de chacun des lots au moment des prélèvements variait entre 240 et $300 \mathrm{~g}$ (tabl. I). Les contenus de cæcums des rats d'un même lot ont été prélevés, mélangés et traités à froid comme nous l'avons décrit précédemment (Combe et ProN, I966). Ils ont ainsi été divisés en 3 fractions :

I : composés solubles dans l'éthanol 80 p. Ioo.

II : composés insolubles dans l'éthanol 80 p. Ioo mais solubles dans l'acide trichloracétique Io p. I0o (ATC).

III : composés insolubles dans l'éthanol et l'ATC ıo p. Ioo.

Les teneurs en azote de différentes fractions ont été déterminées par la méthode de Kjeldahl. Les acides aminés ont été dosés dans les fractions II et III après hydrolyse acide par chromatographie sur colonne (MoORE, SPACKMANN et Stein, I958) sur appareil automatique. 


\section{TABLEAU I}

\begin{tabular}{|c|c|c|c|c|}
\hline Régime nature $(\mathbf{1}) \ldots \ldots \ldots \ldots$ & A & $\mathrm{B}$ & C & $D$ \\
\hline$(\mathrm{N} \times 6,25)$ M. A. p. $100 \quad \ldots \ldots \ldots$ & 22 & 7 & 4 & 0.8 \\
\hline $\begin{array}{l}\text { Rats axéniques : } \\
\text { Nombre } \ldots \ldots \ldots \ldots \ldots \ldots \ldots \\
\text { Poids m. (g) } \ldots \ldots \ldots \ldots \ldots\end{array}$ & $\begin{array}{r}9 \\
270\end{array}$ & $\begin{array}{r}10 \\
240\end{array}$ & $\begin{array}{r}1 / 4 \\
2633\end{array}$ & $\begin{array}{r}5 \\
240\end{array}$ \\
\hline $\begin{array}{l}\text { Rats holoxéniques : } \\
\text { Nombre } \ldots \ldots \ldots \ldots \ldots \ldots \ldots \\
\text { Poids } m \text {. (g) } \ldots \ldots \ldots \ldots \ldots\end{array}$ & $\begin{array}{r}5 \\
276\end{array}$ & $\begin{array}{r}16 \\
240\end{array}$ & $\begin{array}{r}2.2 \\
260\end{array}$ & $\begin{array}{r}10 \\
300\end{array}$ \\
\hline
\end{tabular}

(1) Le régime A contient 22 p. $100 \mathrm{M}$. A., dont 20 p. 100 de caséine et 58 p. 100 de crème de riz, sa composition détaillée a été décrite précédemment (ComBe et al., 1965).

Le régime $\mathrm{B}$ contient $7 \mathrm{p} .100 \mathrm{M}$. A. il a été obtenu en remplaçant la caséine du régime $22 \mathrm{p} .100 \mathrm{M}$. A. par de la crème de riz.

Le régime $\mathrm{C}$ contient \& p. $100 \mathrm{M}$. A. La poudre d'œu est sa seule source d'azote, la crème de riz a été remplacée par de l'amidon de maîs.

Le régime $\mathrm{D}$ contient $0,8 \mathrm{p} .100 \mathrm{M}$. A. (pratiquement protéiprive) il a été obtenu en remplaçant la poudre d'ceuf par de l'amidon de maïs.

TABLEAU 2

Régimes

Composition en acides aminés ( $\mathrm{g} / \mathrm{I} 6 \mathrm{~g} \mathrm{~N})$.

\begin{tabular}{|c|c|c|c|}
\hline Régimes & 22 p. 100 & $\begin{array}{c}\text { B } \\
7 \text { p. } 100, \text { riz }\end{array}$ & $\frac{\mathrm{C}}{\text { \& p. } 100 \text {, œuf }}$ \\
\hline Acide aspartique.... & 7,4 & 9,2 & 10,5 \\
\hline Thréonine ........ & 3,8 & 3,6 & 5,0 \\
\hline Sérine ........... & 5,2 & 5,2 & 7,8 \\
\hline Acide glutamique & 20,85 & 18,2 & 12,6 \\
\hline Proline .......... & $\because, 15$ & 4,65 & 3,7 \\
\hline Glycine ........ & 2,8 & 4,75 & 3,3 \\
\hline Alanine $\ldots \ldots \ldots$ & 3,75 & 6,0 & 5,85 \\
\hline Valine $\ldots \ldots \ldots \ldots$ & 7,0 & 6,85 & 7,3 \\
\hline Cystine .......... & 0,75 & 2,7 & 2,7 \\
\hline Méthionine $\ldots \ldots \ldots$ & 3,35 & 2,7 & 3,3 \\
\hline Isoleucine $\ldots \ldots \ldots$ & 5,25 & 4,6 & 5,65 \\
\hline Leucine ......... & 9,2 & 8,8 & 9,0 \\
\hline Tyrosine $\ldots . . . \ldots$ & 5,1 & 5,05 & 5,0 \\
\hline Phénylalanine ...... & 5,45 & 5,5 & 4,25 \\
\hline Lysine $\ldots \ldots \ldots \ldots$ & 6,9 & 4,1 & 7,5 \\
\hline Histidine.......... & 2,8 & 2,4 & 2,45 \\
\hline Arginine $\ldots \ldots \ldots \ldots$ & 4,5 & 8,7 & 6,3 \\
\hline
\end{tabular}


Les quantités d'azote renfermées dans chaque fraction des contenus de cæcums des animaux ayant reçu les différents régimes sont sur la figure i. Quel que soit le régime, les cæcums des rats axéniques renferment plus d'azote que ceux des rats " holoxéniques "; cette différence est due à une augmentation de l'importance des matières azotées contenues dans les fractions I et II, qui contraste avec une diminution de celle des matières azotées de la fraction III liée certainement à l'absence de corps microbiens et peut-être à la diminution de la vitesse de desquamation de l'épithélium intestinal. Chez les animaux axéniques, les plus grandes différences de teneurs en azote liées au régime sont observées dans le cas de la fraction I et les plus faibles dans le cas de la fraction III. De plus les fractions II et III des contenus de cæcums des rats qui ont reçu le régime B (7 p. I oo M.A, riz) renferment respectivement autant et plus d'azote que les fractions correspondantes provenant des animaux qui ont consommé le régime A (22 p. Ioo MA, caséine).

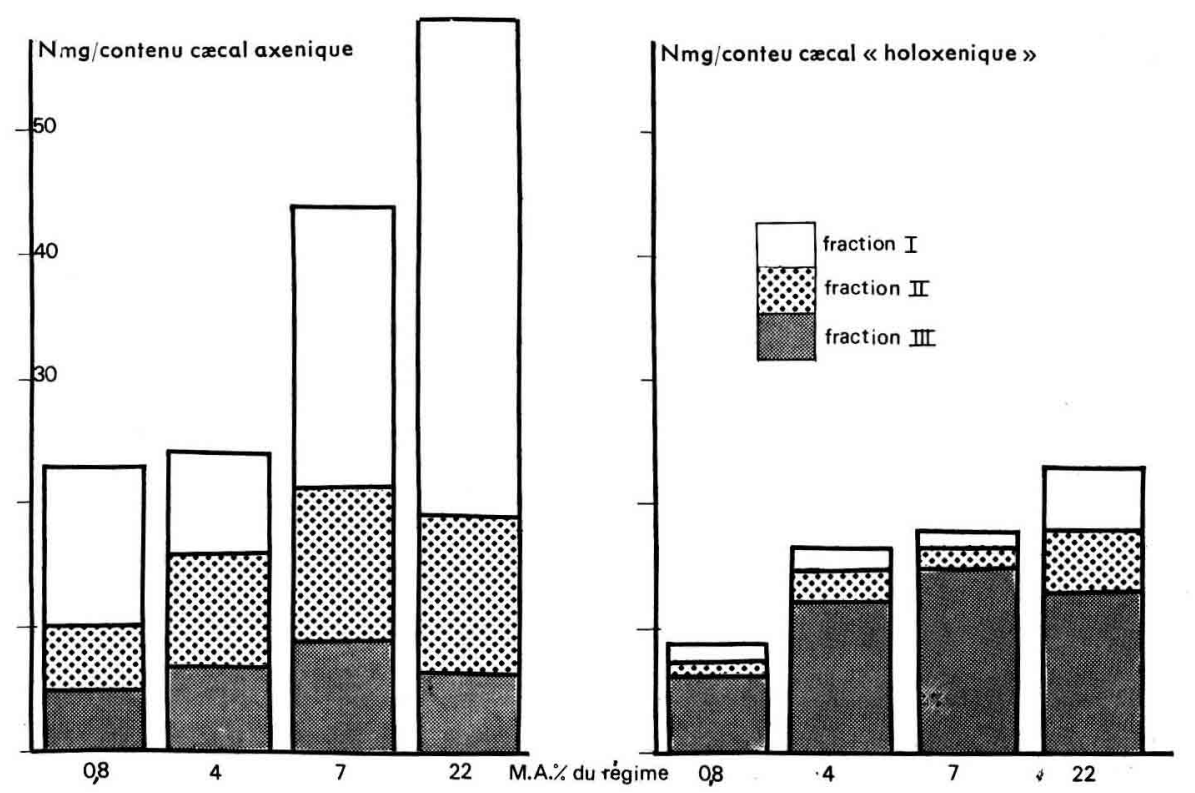

FIg. I. - Azote contenu dans le cấcum de rat axénique et "holoxénique ", répartition selon la solubilité dans l'éthanol 80 p. 100 et l'A. T. C. 10 p. 100. Variation avec la tencur en matières azotées du régime

Les résultats de l'étude des composés azotés solubles dans l'éthanol ont été publiés précédemment (СомBE et al., I965; CомBE et SACQUET, I966); les acides aminés libres sont présents en grandes quantités : 5,2 et 3,7 mg/g de contenus frais respectivement pour les régimes à 22 p. Ioo et à $7 \mathrm{p}$. I oo de protéines, dans le cas des rats axéniques, alors que leur concentration est très faible dans le cas des " holoxéniques": $0,5 \mathrm{et} 0,2 \mathrm{mg} / \mathrm{g}$ respectivement pour les deux régimes. De plus, leur répartition n'est pas la même pour les deux types d'animaux. C'est ainsi que les proportions de sérine et de thréonine sont 2 et 4 fois plus élevées, et la proportion d'alanine 2 fois plus faible chez las animaux axéniques que chez les témoins. En outre, les cæcums des animaux axéniques contiennent de l'urée en concentration égale à celle du plasma sanguin, alors qu'il n'y en a aucune trace dans le cas des holoxéniques.

La composition en acides aminés de la fraction II du contenu de cacum de rat axénique est donné dans le tableau 3.

Les caractéristiques principales de cas composés ont déjà été soulignées dans le cas du régime à 22 p. roo de protéines (COMBE et PION 1966) : les teneurs en thréonine et en sérine sont supérieures, et les teneurs en glycine, leucine, tyrosine, phénylalanine et méthionine inférieures à celles trouvées généralement pour ces acides aminés dans les protéines animales (muscle, tissu intestinal) mais elles rappellent celles des mucoprotéines et de certaines enzymes pancréatiques.

La détermination de la composition en acides aminés de la fraction II des contenus de cæcums de rats axéniques soumis aux autres régimes fait apparaître des caractéristiques similaires. Toutefois, l'influence du régime alimentaire est notable: les teneurs en sérine, valine, isoleucine, leucine 
sont nettement plus élevées dans le cas des rats recevant le régime A (caséine) que dans celui des autres animaux. Les teneurs en acide aspartique, acide glutamique, lysine et histidine diminuent avec le taux protéique de la ration. Il est difficile de dire si ces différences correspondent à des proportions différentes des protéines d'origine alimentaire dans les contenus ou à des variations dans les proportions des matières azotées d'origine endogène. En revanche, la nette augmentation de la teneur en thréonine observée lorsque le taux protéique de la ration diminuc permet de supposer que la proportion des matières azotées d'origine endogène dans les matières azotées totales varie en sens inverse de l'apport azoté alimentaire. De plus, la part des acides aminés dans les matières azotées totales de la fraction II est nettement plus importante dans le cas du régime à 22 p. Ioo de protéines (64 p. Ioo de l'azote total) que de celui à 7 p. Ioo (52 p. roo de l'azote total) et des autres régimes (4o et $47 \mathrm{p}$. I 00 de l'azote total). Il est vraisemblable que cette augmentation est liée à une diminution de la proportion de sucres aminés caractéristiques d'une partie des matières azotées endogènes.

\section{TABLEAU 3}

Contenus de cacum de rat axénique, fractions $I I$ et III

Composition en acides aminés $(g / 16 \mathrm{~g} N)$. Variations avec le régime

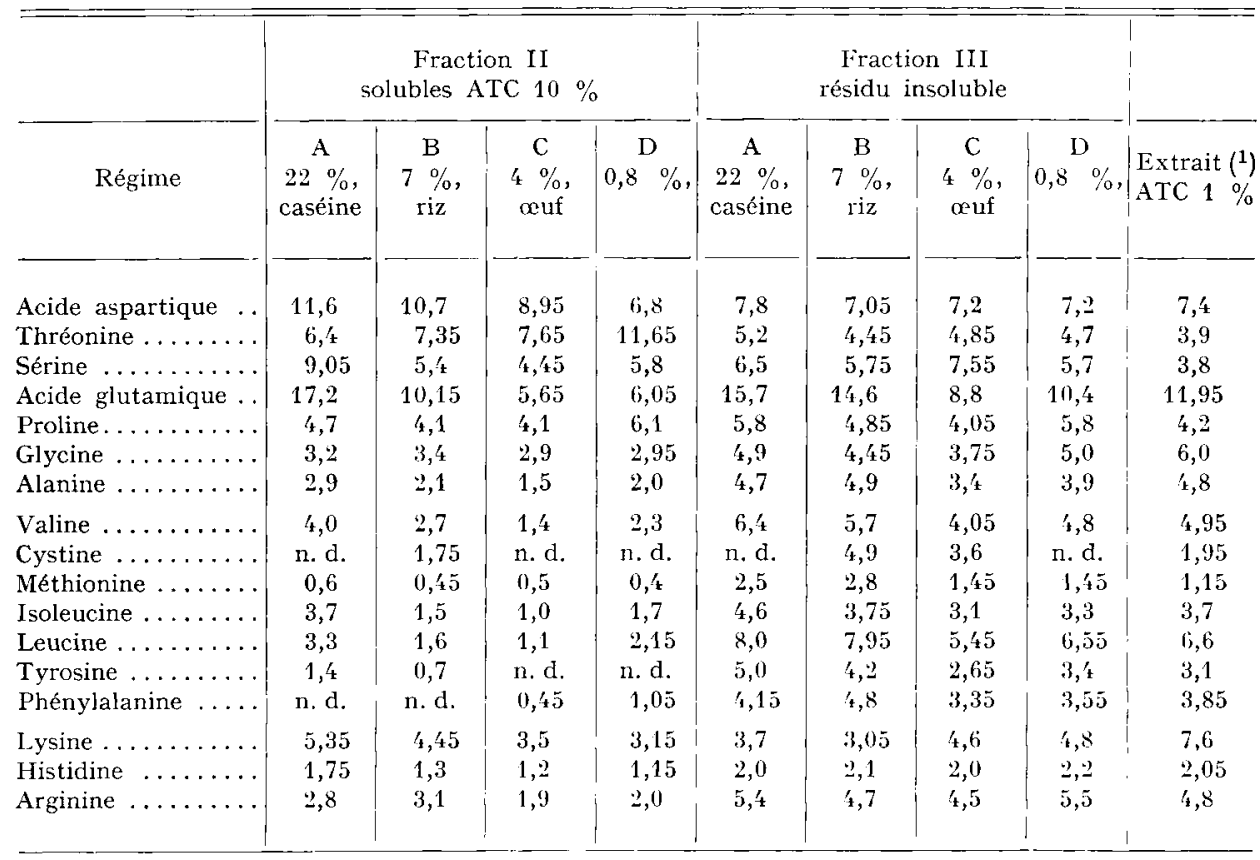

(1) Extrait provenant du grattage de l'épithélium intestinal (porc).

Les concentrations en acides aminés indispensables sont généralement plus faibles dans le cas du régime $\mathrm{C}$ (4 p. Ioo, œuf) que dans celui du régime $\mathrm{D}(0,8 \mathrm{p}$. Ioo : protéiprive). Toutefois, comme l'importance de cette fraction est plus grande chez les animaux ayant consommé la ration à base de protéines d'œuf, les quantités de la plupart de ces acides aminés présentes dans les fractions II des cæcums sont du même ordre de grandeur pour les deux régimes. La lysine et l'histidine se trouvent en quantités plus importantes dans les cæecums des rats ayant reçu le régime $\mathrm{C}$ (4 p. IOo, œuf).

Les proportions des différents acides aminés de la fraction III varient relativement peu avec le régime, et ne diffèrent pas beaucoup de celles des aliments ingérés, ni de celles des protéines extraites de l'épithélium intestinal (tabl. 3). Les variations observées ne reflètent que faiblement celles de la composition des aliments : la teneur en acide glutamique des protéines de la fraction III des contenus de cæcums varie nettement moins que la teneur correspondante des protéines alimentaires. 
Les teneurs en lysine des protéines des contenus sont nettement plus faibles que celles des protéines épithéliales et des protéines alimentaires, même dans le cas du riz. Elles diminuent quand la teneur en protéine de l'aliment augmente. Ces faibles teneurs sont probablement dues à la libération préférentielle de lysine par des enzymes protéolytiques d'origine pancréatique, ou provenant de la muqueuse intestinale. En effet, Reddy, Pleasants et Wostmann (I969) ont trouvé que les enzymes pancréatiques et en particulier la trypsine avaient une activité très élevée dans le cæcum de rats axéniques. De plus, Jervis et Biggers (1964) ont mis en évidence chez la souris axénique la présence en concentrations particulièrement importantes dans la muqueuse intestinale, d'endo-enzymes qui doivent être libérées au cours de la desquamation de ce tissu.

La teneur en sérine reflète, dans le cas de l'œuf, celle de la protéine ingérée. Les tencurs en cystine se sont révélées, dans les cas où elles ont été déterminées, très élevées et notablement différentes de celles des aliments.

On observe peu de différence de composition entre les contenus des animaux ayant reçu le régime protéiprive, et ceux qui ont consommé le régime à 4 p. Ioo de protéines d'œuf. Comme les variations de la quantité d'azote présente dans la fraction III sont très faibles par rapport aux différences des quantités d'azote ingérées, les quantités de chacun des acides aminés présents dans chaque contenu varient très peu en comparaison des différences de teneurs azotées dans les aliments. Elles sont toutefois nettement plus élevées chez les animaux qui ont reçu le régime $\mathrm{C}$ ( 7 p. Ioo, riz) que chez les autres, en particulier en ce qui concerne l'acide glutamique, la phénylalanine, l'alanine, la tyrosine, la leucine, la valine et l'isoleucine (tabl. 2 et 3 ).

Cette relative accumulation doit être le résultat d'une indigestibilité d'une partie des matières azotées du riz, peut-être liée à la stérilisation de l'aliment par la chaleur alors que les faibles quantités d'acides aminés trouvées dans les matières azotées insolubles des contenus de cæcums des animaux ayant consommé les autres rations, et en particulier le régime le plus riche en azote, permettent de supposer que la digestibilité vraie de leurs protéines est très élevée.

Reçu pour publication en juillet $19 \% 0$.

\section{SUMMARY}

\section{EFFECT OF NATURE AND RATE OF DIETARY PROTEINS ON THE AMINO-ACID COMPOSITION OF THE CECAL, CONTENT OF GERM-FREE RATS}

The purpose of this work was to study nitrogenous matter in cecal contents of germfree rats under several dietary conditions. Germfree $(\mathrm{GF})$ and Conventional (CV) adult male Wistar (CF), Gif) rats were fed ad libitum according to the procedure described table $I$ and 2 for 8 days. At the end of the experiment, cecal contents from numbutal anesthetized rats of the same group were pooled together. Fractionation from each batch gave : I = ethanol 80 p. roo soluble material ; II $=$ TCA Io p. Ioo soluble ; III = residue insoluble in ethanol and TCA. Nitrogen (micro kjeldahl) and amino acids (automatic column chromatography according to Moore and STEIN after acid hydrolysis).

In each fraction were assayed. Dry matter intake was $20 \mathrm{~g} /$ day/animal before sampling day. In these experimental conditions the GF cecums hold more nitrogen than the CV (fig. I), and this difference accounts for ethanol and TCA soluble fractions (I and II). GF fractions I and II Nitrogen contents increase with level of crude protein in the diet, while fracticn III Nitrogen content is rather constant. $\mathrm{B}$ diet leads to a higher nitrogen content for fraction III in GF and $\mathrm{CV}$ pools. GF fraction II (table 3) is always higher in threonine $(6.4 ; 7.35 ; 7.65$; I $1.65 \mathrm{mg} / \mathrm{I} 6 \mathrm{~g} \mathrm{~N}$ with respectively $\mathrm{A}, \mathrm{B}, \mathrm{C}$, D diets) and serine $(9.05 ; 5.4 ; 4.45 ; 5.8 \mathrm{mg} / \mathrm{I} 6 \mathrm{~g} \mathrm{~N}$ with $\mathrm{A}, \mathrm{B}, \mathrm{C}, \mathrm{D}$ diets) and lower in glycine leucinc tyrosine phenylalanine and methionine than usual for animal proteins. Mucoproteins and pancreatic enzymes are known to have similar characteristics. When crude protein of the diet falls from $22 \mathrm{p}$. Ioo to 7,4 and $0.8 \mathrm{p}$. Ioo, threonine concentration in the GF fraction II pools increases very much, and total amino acids nitrogen accounts for less of the total nitrogen, that is aminosugars proportions become higher. These observations lead to speculate that proportion of endogenous proteins of GF cecal proteins increases when crude proteins proportion decreases in the diet. In fraction III (table 3 ) of GF cecal content pools. amino acid composition was not found different from amino acid composition of intestinal epithelium. Amino acid levels in fraction III were not modified by crude proteins of 
the diet except for the diet $\mathrm{C}$ where steam sterilization might have been of some dommage for rice proteins. Lysine only was found lower in all fractions III than in animal proteins : 3.7 ; $3.05 ; 4.6 ; 4.8 \mathrm{mg} / \mathrm{I} 6 \mathrm{~g} \mathrm{~N}$ with $\mathrm{A}, \mathrm{B}, \mathrm{C}, \mathrm{D}$ diets. This fact may be accounted for specific action of pancreatic enzymes on lysine bounds proteins of the germfree digestive contents.

\section{RÉFÉRENCES BIBLIOGRAPHIQUES}

Combe E., Penot E., Charlier H., Sacouet E., ig65. Métabolisme du rat "germfree ". Teneurs des contenus digestifs en certains composés azotés, en sodium et en potassium. Teneurs de quelques tissus en acides nucléiques. Ann. Biol. anim. Bioch. Biophys., 5 (2), 189-206.

Сомве E., Pion R., ig66. Note sur la composition en acides aminés du contenu de cæcum de rats axéniques et de rats témoins. Ann. Biol. anim. Bioch. Biophys., 6 (2), 255-259.

Combe E., SAcquet E., Ig66. Influence de l'état axénique sur divers composés azotés contenus dans le cacum de rats albinos recevant des quantités variables de protéines. C. $R$. Acad. Soc. Paris, Ser. D, 262, $685-688$.

Creamer B., I967. The turnover of the epithelium of the small intestine. Br. med. Bull., 23 (3), 226-230.

Jervis H. R., BigGers D. C., I964. Mucosal enzymes in the cæcum of conventional and germfree mice. Anat. Rec.,, 148 (4), 59I-597.

Moore S., Spackmann D. M., Stein W. M., i958. Chromatography of amino acids on sulfonated polystyrene resins. Anal. Chem., 30, I I85-I I90.

Raibaud P., Dickinson A. B., Sacouet E., Charlier H., Mocouot G., ig66. La microflore du tube digestif du rat. IV. Implantation contrôlée chez le rat gnotobiotique de différents genres microbienc isolés du rat conventionnel. Ann. Inst. Pasteur, 111, 193-2 Io.

Reddy B.S., Pleasants J. R., Wostmann B.S., ig69. Pancreatic enzymes in germ free and conventional rats fed chemically defined, water-soluble diet free from natural substrates. J. Nutr., 97 (4), $327-334$.

Twombly J., Meyer J. H., r96r. Endogenous nitrogen secretions into the digestive tract. J. Nutr., 74, $453-460$.

Twombly-SNook J., r965. Dietary regulation of pancreatic enzyme synthesis, secretion and inactivation in the rat. J. Nutr., 87, 297-305. 